\title{
Numerical Simulation of the Unsteady Cavitation Behavior of an Inducer Blade Cascade
}

\author{
Olivier Coutier-Delgosha, ${ }^{*}$ Yannick Courtot, ${ }^{\dagger}$ Florence Joussellin,,${ }^{\ddagger}$ and Jean-Luc Reboud ${ }^{\S}$ \\ Institut National Polytechnique de Grenoble, 38041 Grenoble Cedex 9, France
}

\begin{abstract}
One source of unsteadiness in turbopump inducers consists in a rotating cavitation behavior, characterized by different cavity shapes on the different blades, which leads to super- or subsynchronous disturbances. This phenomenon is simulated for the case of a simple two-dimensional blade cascade corresponding to a typical fourblade inducer. A numerical model of unsteady cavitating flows was adapted to take into account nonmatching connections and periodicity conditions. Single-channel and four-channel computations were performed, and in the latter case, nonsymetrical unstable flow patterns were obtained. Limits of stability according to the mass flow rate and the cavitation number are presented. Qualitative comparisons with experiments, instability criterion, and the mechanisms of instabilities are also investigated.
\end{abstract}

\begin{tabular}{|c|c|}
\hline \multicolumn{2}{|r|}{ Nomenclature } \\
\hline$A_{\min }$ & $\begin{array}{l}=\text { minimum speed of sound in the two-phase } \\
\text { mixture, } \mathrm{m} / \mathrm{s}\end{array}$ \\
\hline $\boldsymbol{C}\left(\boldsymbol{C}_{m}, \boldsymbol{C}_{u}\right)$ & $=$ velocity vector in fixed frame, $\mathrm{m} / \mathrm{s}$ \\
\hline$C_{p}$ & $\begin{aligned}= & \text { dimensionless static pressure } \\
& \left(p-p_{\text {ref }}\right) /\left(\frac{1}{2} \rho U^{2}\right)\end{aligned}$ \\
\hline$P$ & $=$ total pressure, $P+\frac{1}{2} \rho C^{2}, \mathrm{~Pa}$ \\
\hline$P_{1}, P_{2}$ & $=$ total pressure at inlet and outlet, $\mathrm{Pa}$ \\
\hline$p$ & $=$ local static pressure, $\mathrm{Pa}$ \\
\hline$p_{\text {ref }}, p_{\text {vap }}$ & $\begin{aligned}= & \text { reference pressure (inlet pressure), } \\
& \text { vapor pressure, } \mathrm{Pa}\end{aligned}$ \\
\hline$p_{1}, p_{2}$ & $=$ static pressure at inlet and outlet, $\mathrm{Pa}$ \\
\hline$r, R_{c}, R$ & $\begin{array}{l}=\text { inducer radius, radius corresponding } \\
\text { to the blade cascade, tip radius, } m\end{array}$ \\
\hline$S$ & $=$ nondimensional surface of a grid cell \\
\hline$S_{\text {flow }}$ & $=$ cross section of the blade-to-blade channel, $\mathrm{m}^{2}$ \\
\hline$T_{\text {ref }}$ & $\begin{array}{l}=\text { time corresponding to the passage of one blade } \\
\text { in the fixed frame, } \mathrm{s}\end{array}$ \\
\hline$U$ & $=$ training velocity at inducer radius $R_{c}, R_{c} \Omega, \mathrm{m} / \mathrm{s}$ \\
\hline $\boldsymbol{W}\left(\boldsymbol{W}_{m}, \boldsymbol{W}_{u}\right)$ & $=$ relative velocity vector, $\mathrm{m} / \mathrm{s}$ \\
\hline$\alpha$ & flow incidence at the blade leading edge, rad \\
\hline$\alpha_{v}$ & $=$ local void fraction \\
\hline$\rho_{l}, \rho_{v}, \rho$ & $\begin{array}{l}=\text { nondimensional density of the liquid, } \\
\text { of the vapor, of the mixture }\end{array}$ \\
\hline$\rho_{\text {ref }}$ & $=$ reference density $\rho_{l}$ \\
\hline & $=$ cavitation number, $\left(p_{1}-p_{\text {vap }}\right) /\left(\frac{1}{2} \rho U^{2}\right)$ \\
\hline$\Phi, \Phi_{\text {ref }}$ & $\begin{array}{l}=\text { flow coefficient, } \boldsymbol{C}_{m} /(U) \text {, and reference } \\
\text { flow coefficient }\end{array}$ \\
\hline
\end{tabular}

*Ph.D. Student, Turbomachines/Cavitation, Laboratoire des Ecoulements Géophysiques et Industriels; currently Graduate Research Assistant, Ecoulements Diphasiques et Turbulences, Laboratoire des Ecoulements Géophysiques et Industriels, B.P. 53; olivier.coutier-delgosha@ @mg.inpg.fr.

${ }^{\dagger}$ Ph.D. Student, Turbomachines/Cavitation, Laboratoire des Ecoulements Géophysiques et Industriels; currently Engineer, Snecma Moteurs, B.P. 81, 91003 Evry Cedex, France; yannick.courtot@ snecma.fr.

¥Assistant Professor, Turbomachines/Cavitation, Laboratoire des Ecoulements Géophysiques et Industriels, B.P. 53; florence.joussellin@hmg. inpg.fr.

${ }^{8}$ Professor, Turbomachines/Cavitation, Laboratoire des Ecoulements Géophysiques et Industriels; currently Professor, Université Joseph Fourier, B.P. 166, 25 avenue des Martyrs, 38042 Grenoble Cedex 9, France; jeanluc.reboud@grenoble.cnrs.fr.
$\Psi, \Psi_{\text {ref }}$
$=$ head coefficient $\left(P_{2}-P_{1}\right) /\left(\rho_{l} U^{2}\right)$, head coefficient at cavitation inception
$\Omega$ $=$ inducer angular velocity, $\mathrm{rad} / \mathrm{s}$

\section{Introduction}

$\mathbf{T}$ $\mathrm{O}$ achieve operating at high rotational speed and low inlet pressure, rocket engine turbopumps are generally equipped with an axial inducer stage working in cavitating conditions (Fig. 1). Cavitation develops on suction side of the blades and at inducer periphery near the tip. Peripheral cavitation concerns all cavitating structures that appear near the shroud casing at the inducer inlet, as backflow of the pump ${ }^{1}$ and cavitating tip vortices. ${ }^{2}$ The presence of vapor induces disturbances that can result in substantial performance losses. Moreover, when inlet pressure is decreased from cavitation inception to breakdown of the pump, unsteady phenomena may appear, associated with different blade cavitation patterns. Experimental results point out two main types of cavitation instabilities: a self-oscillation behavior of cavitation sheets, whose mechanism was studied in cavitation tunnels and analyzed in detail by many authors, ${ }^{3-5}$ and a rotating cavitation behavior, mainly observed in inducers, which shows different sizes of cavitation structures in the different blade-to-blade passages of the machine and leading to super- or subsynchronous perturbations.

This last phenomenon strongly depends on the cavitation development in the machine. A typical example is given in Fig. 2: At cavitation inception, only a steady and balanced flow pattern with one short attached cavity on each blade is observed from flow visualizations. When the cavitation parameter is slightly decreased, a steady and alternate cavitating configuration appears (only on four blade inducers) with alternatively one short and one long cavity. For a lower cavitation parameter, ${ }^{6}$ just above breakdown, an unsteady flow pattern called rotating cavitation can be identified: Unbalanced attached cavities are observed in the different channels, their distribution rotating faster than the inducer, ${ }^{7,8}$ which leads to large radial loads on the shaft. ${ }^{9}$ Finally, near the breakdown of the inducer, a steady and balanced flow pattern with fully developed cavitation is observed.

These instabilities induce some strong radial forces that may perturb the rotor balance and important pressure fluctuations in the lines. They must be quantified and controlled to avoid any major effect on the global pump behavior.

Over the past few years, numerical models have been developed to predict the cavitation instabilities in inducers. They are based on stability analyses and linear approach and take into account the total flow rate variations through a cavitating blade-to-blade channel, ${ }^{10,11}$ or calculate the flow around attached cavities. ${ }^{12,13}$ To improve the understanding and the prediction capability of cavitation instabilities, numerical and experimental analyses are carried out in France through collaborations between the Laboratoire des Ecoulements Géophysiques et Industriels, the Rocket Engine Division of 


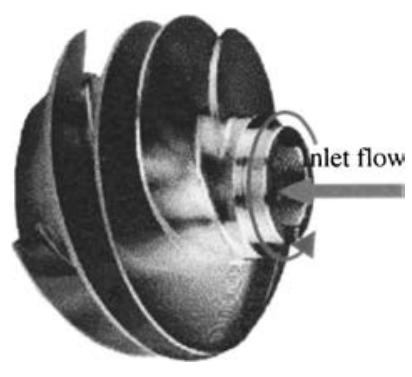

a) Side view of the geometry

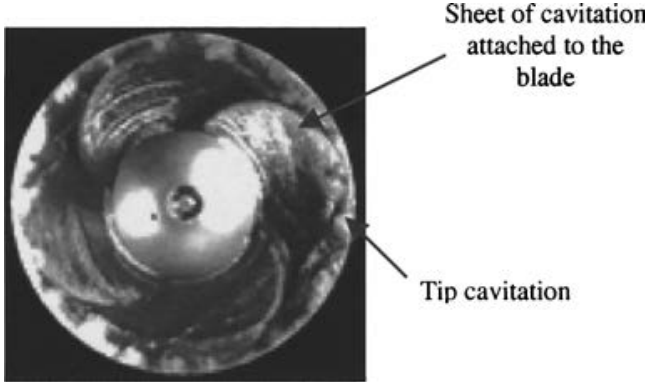

b) Front view in cavitating conditions

Fig. 1 Rocket engine four-blade turbopump inducer.

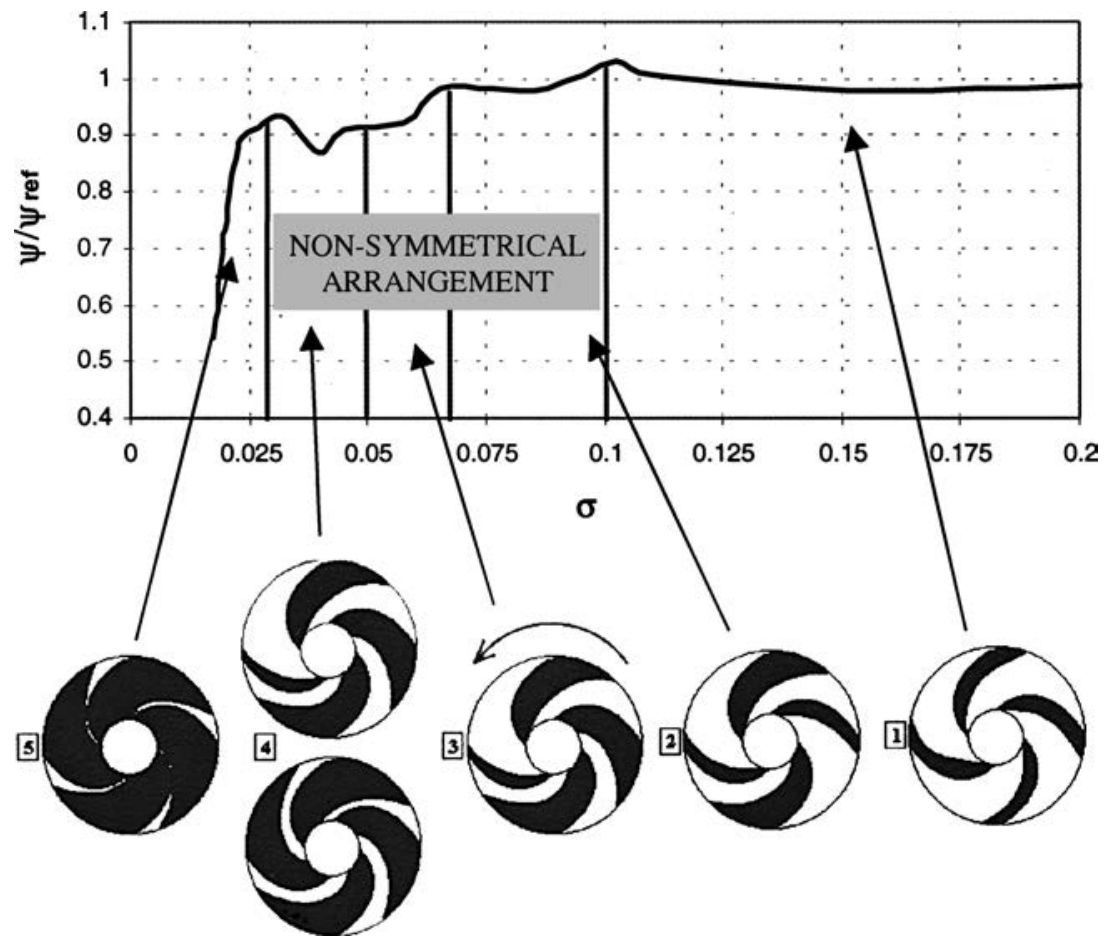

Fig. 2 Cavitation patterns and performance evolution as the cavitation number decreases in four-blade inducer. ${ }^{7}$

SNECMA Moteurs and the French space agency Centre National d'Etudes Spatiales. A two-dimensional model was developed ${ }^{14-16}$ to simulate unsteady cavitation phenomena in inducers, such as pulsating cavities or vapor cloud shedding. The liquid/vapor mixture is considered in this model as a single fluid whose density varies from the liquid density to the vapor density, with respect to the local static pressure. The model has been validated on numerous cases, such as venturi-type sections or hydrofoils in cavitation tunnels, and the results demonstrated a good agreement with experiments. Its specificity is a reliable simulation of the cyclic behavior of self-oscillating cavities. $^{16,17}$

In the present paper, the numerical model is applied to the simulation of the other instability observed in inducers, namely, the nonsymmetrical cavitation pattern. This first attempt is performed in a two-dimensional blade cascade corresponding to a four-blade inducer. The main objective was to take into account the interaction phenomena between the different blade-to-blade passages and to evaluate their effect on the unsteady behavior of the cavitation sheets on each blade. We focus in the present study on the ability of the numerical model to distinguish stable configurations from unstable ones, with a special attention paid to the mechanisms of instabilities.

The computational domain is the blade-to-blade geometry, that is, a $(m, r \theta)$ representation, where $m$ is the meridian coordinate, $r$ the radius (here constant), and $\theta$ the revolution angle. The twodimensional blade-to-blade channels were drawn by cutting the three-dimensional inducer geometry at constant radius $R_{c}$ equal to $80 \%$ of the tip radius $R$ (Fig. 3 ). The computational grids of the four channels must be identical to ensure a precise detection of unstable cavitating behaviors. Indeed, different grids would induce spurious numerical errors that could be held responsible for the appearance of instability in the flowfield. Unfortunately, this condition cannot be imposed with a single mesh applied to the whole geometry because of the orthogonality of the cells that is required by the numerical model in the computational domain. Therefore, four identical separate grids are used for the four channels with three connections and one periodicity condition. It can be seen in Fig. 4 that it is quite impossible to obtain matching cells at these boundaries because of their curvature due to the high inclination of the blades in the $(m, r \theta)$ representation. Therefore, a new method to treat nonmatching boundaries is presented in this paper. It is based on an interpolation technique that guarantees, through its integration inside the algorithm, both mass and momentum conservation.

Computations were performed with different values of the cavitation number $\sigma$, to point out stable or unstable cavitating flows. Our present objective is to demonstrate the capability of the numerical model to simulate the mechanisms of nonsymmetrical flow arrangements. Results of the calculations are also compared to experimental observations performed previously at the Centre de Recherches et d'Essais de Machines Hydrauliques de Grenoble (CREMHyG) laboratory.

\section{Physical Model}

The cavitation model is based on a single-phase flow approach, which assumes that only one fluid is considered. ${ }^{18}$ This fluid is 

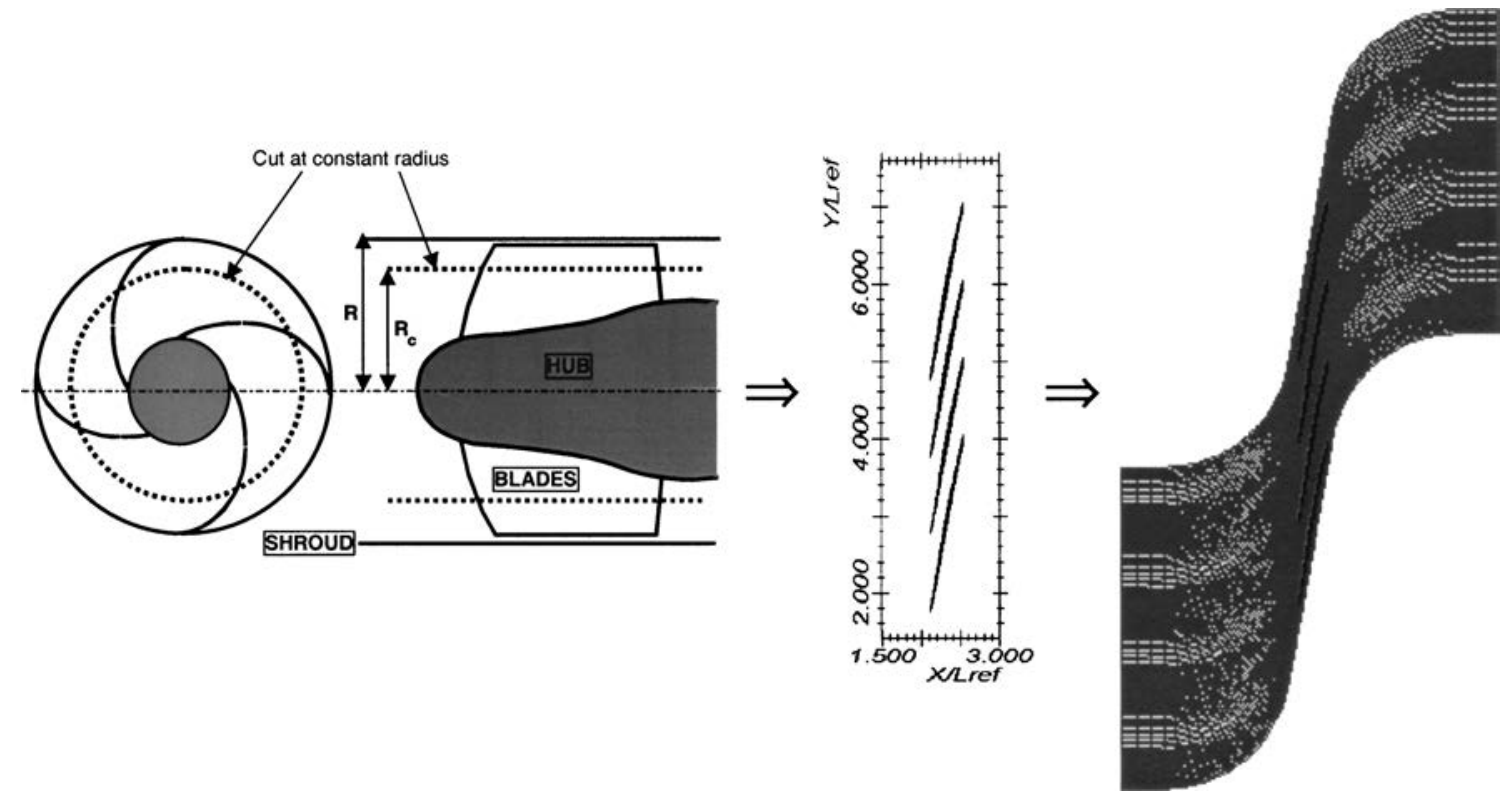

Fig. 3 Passage from three-dimensional real geometry to blade cascade.

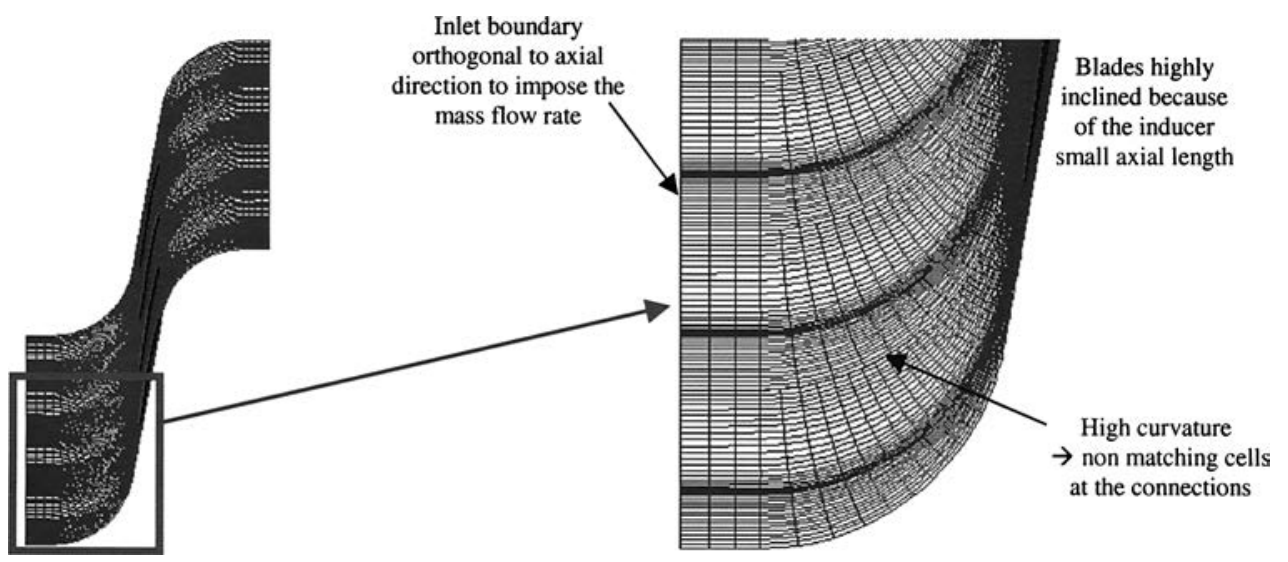

Fig. 4 Nonmatching cells at the connections.

characterized by a density $\rho$ that varies in the computational domain with respect to a state law. When the density in a cell equals the liquid density $\rho_{l}$, all of this cell is occupied by liquid, and if it equals the vapor density $\rho_{v}$, the cell is full of vapor. Between these two extreme values, the cell is occupied by a water/vapor mixture that we still consider as one single fluid. The void fraction $\alpha_{v}=\left(\rho-\rho_{l}\right) /\left(\rho_{v}-\rho_{l}\right)$ can be defined as the local ratio of vapor contained in this mixture. If the cell is full of vapor, then $\alpha_{v}=1$. However, if a cell is totally occupied by liquid, $\alpha_{v}=0$.

Through this simple model, linking the void ratio $\alpha_{v}$ to the state law, we implicitly treat the fluxes between the phases, without the supplementary assumptions required in the case of a two-phase model.

With regard to the momentum fluxes, our model assumes that locally (in each cell) velocities are the same for liquid and for vapor: In the mixture regions, gas structures are supposed to be perfectly carried along by the main flow. (The friction forces are high, compared to the buoyancy forces.) That hypothesis is often assessed for this problem of sheet-cavity flows, in which the interface is considered to be in dynamic equilibrium. ${ }^{19,20}$ The momentum transfers between the phases are, thus, directly linked to the mass transfers.

Vaporization and condensation processes are managed by a postulated barotropic state law that links the density to the local static pressure. The fluid is supposed to be purely liquid or purely vapor when the pressure is higher or lower than the vapor pressure, respectively. The two cases are joined smoothly in the vapor-pressure neighborhood. It results in the state law presented in Fig. 5, whose

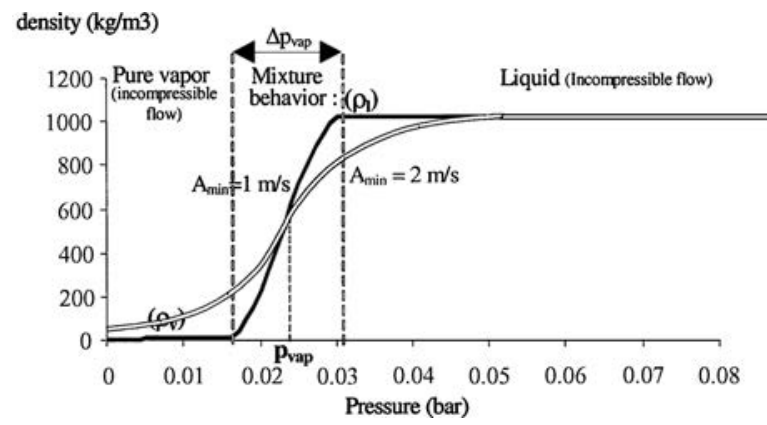

Fig. 5 Barotropic state law $\rho(P)$.

only parameter is the maximum slope $1 / A_{\min }^{2}$, where $A_{\min }^{2}=\partial p / \partial \rho$. $A_{\text {min }}$ can, thus, be interpreted as the minimum speed of sound in the mixture. Its calibration was performed in previous studies ${ }^{15,16}$ in the case of unsteady self-oscillation behavior of sheet cavitation. The optimal value was found to be independent of the hydrodynamic conditions and is about $2 \mathrm{~m} / \mathrm{s}$ for cold water, with $p_{\text {vap }}=0.023 \mathrm{bar}$, and corresponds to $\Delta p_{\text {vap }} \approx 0.06$ bar (Fig. 5). The use of this state law implies that no delay to vaporization or condensation can be considered: Density directly depends on the pressure. Nevertheless, other models including this physical feature ${ }^{20}$ have been also implemented and tested. If the vaporization/condensation terms are correctly tuned, that is, the delay parameters are optimized, then very similar results are obtained. In the present case, we try to simulate 
large-scale fluctuations of the whole cavitation sheets attached to the blades. To obtain numerically smooth cavities, we use a nondimensional ratio $A_{\min } /\left(R_{c} \Omega\right)$ of 0.1 . Rotation speed corresponding to a celerity of sound of $2 \mathrm{~m} / \mathrm{s}$ is then lower than the experimental value.

The main numerical problem of our single-fluid approach results from the difficulty to manage different flow behaviors: incompressible flow in the areas containing pure liquid or pure vapor and a highly compressible flow in the areas of transition between liquid and vapor.

\section{Numerical Model}

To solve the time-dependant Reynolds-averaged Navier-Stokes equations associated with the barotropic state law presented earlier, the numerical code applies the SIMPLE algorithm on twodimensional structured curvilinear-orthogonal meshes, with modifications to take into account the cavitation process. The details of the numerical resolution were presented by Coutier-Delgosha et al. ${ }^{16}$ It is based on an implicit method for the time discretization, and the HLPA nonoscillatory, second-order convection scheme proposed by Zhu. ${ }^{21}$ A complete validation of the method was performed, and the influence of the numerical parameters was widely investigated. The results are reported in Ref. 16.

A standard $k$-epsilon RNG model of turbulence is used. This model does not allow any simulation of the self-oscillation behavior of sheet cavitation, as reported by Coutier-Delgosha et al. ${ }^{22}$ The effects of the two-phase mixture compressibility on the turbulence structure must be taken into account to simulate this phenomenon correctly. A modification of the standard model was proposed in Refs. 15 and 16 to solve this problem efficiently. In the present study, only the rotating cavitation pattern is investigated. This is a first attempt to predict numerically the cavitating coupling between the channels, and so the interaction of its mechanisms with the self-oscillation of each cavity is not suitable. Thus, the standard turbulence model is applied.

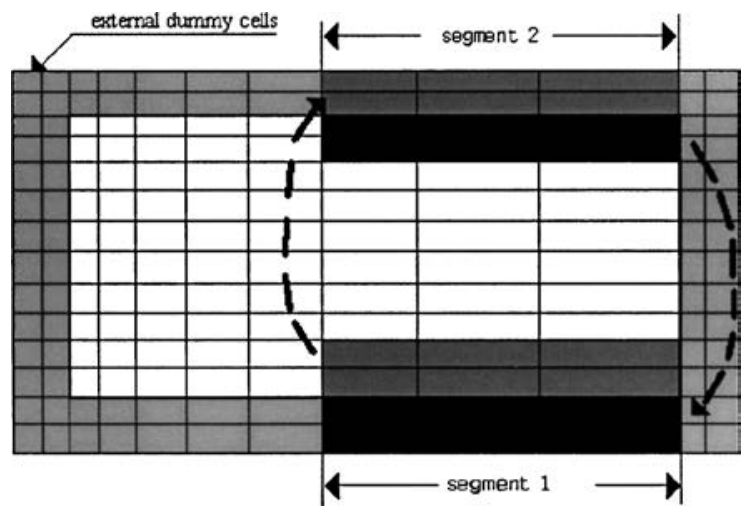

a)

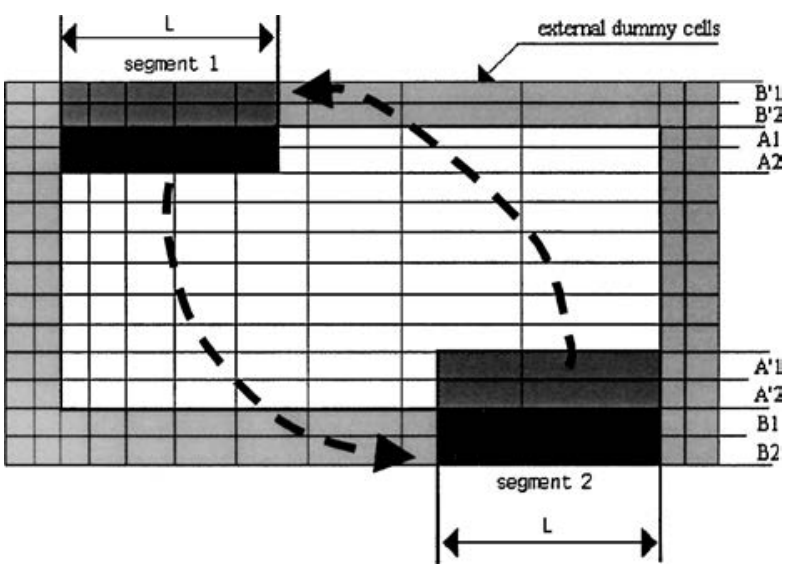

b)

Fig. 6 Information transfer between segments 1 and 2 in case of a) matching and b) nonmatching boundaries.

\section{Connection of Nonmatching Boundaries}

The boundary condition setting is based on two rows of dummy cells generated around the computational domain. Connections or periodicity conditions between two frontiers are obtained by transferring variables from inner cells to dummy cells. In matching boundary cases (identical grids on the two sides of the frontier, Fig. 6a), no interpolation is necessary, and the procedure involves no supplementary numerical error. In nonmatching boundary cases (Fig. 6b), special care must be paid to constrict the errors introduced by the interpolations and to respect the conservative character of the resolution. Indeed, the spurious generation of mass or momentum inside the domain, through connections or periodicities, would be very prejudicial to the rate of convergence.

We present here the general features of the process indicated in Fig. 6b. It consists in transferring the information from rows A1 and $\mathrm{A} 2$ to rows $\mathrm{B} 1$ and $\mathrm{B} 2$ and from rows $\mathrm{A}^{\prime} 1$ and $\mathrm{A}^{\prime} 2$ to rows $\mathrm{B}^{\prime} 1$ and $\mathrm{B}^{\prime} 2$.

\section{A. Geometry of Dummy Cells}

In the case of connections or periodicity conditions, the shape of the dummy cells is of first importance because their width, their length, and their curvature strongly affect the computation. Any geometrical difference between the dummy cells and the inner corresponding ones would enhance the numerical errors by creating some spurious mass and momentum flow rates. Therefore, these cells must be as similar as possible to the cells of the original corresponding row.

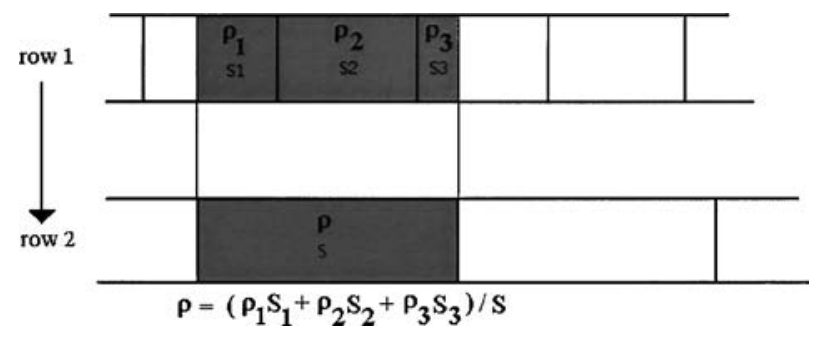

Fig. 7 Density transfer

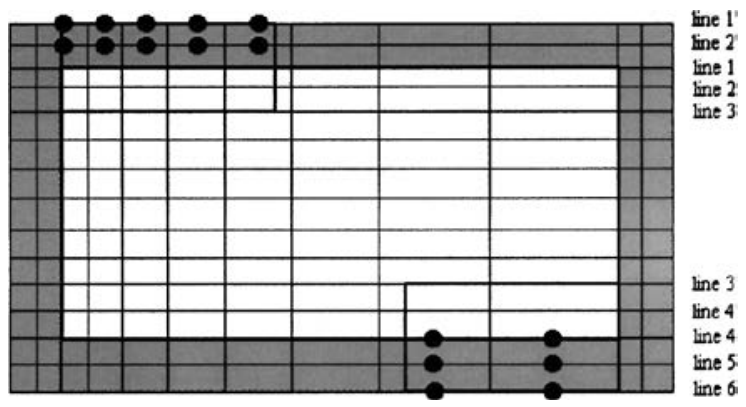

a)

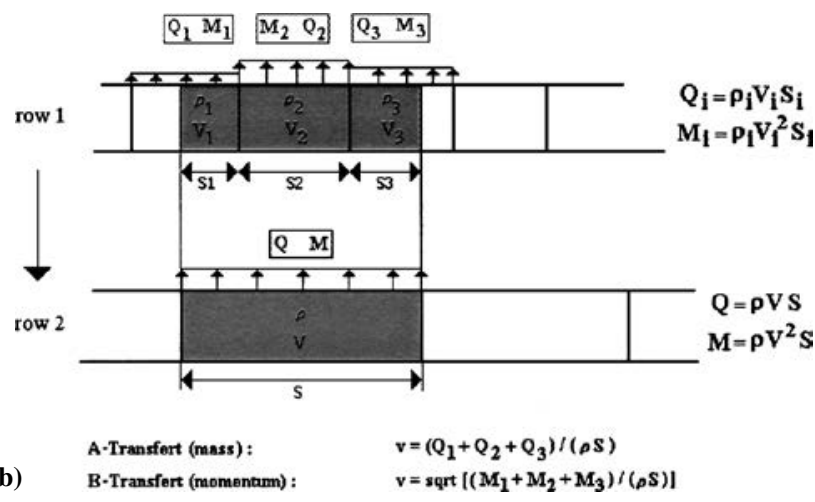

Fig. 8 Transfer of the velocity component $v$ : a) updated velocities $v$ in the dummy cells and $b$ ) alternate conservation of mass and momentum. 


\section{B. Interpolations of Variables}

All variables are transported from the computational domain to the dummy cells, that is, from A rows to B rows, through interpolations. The kind of interpolation depends on the transmitted variable. The transfer aims to ensure the conservation of both mass and momentum. In other words, the mass quantity passing through one of the two frontiers must be as close as possible to the mass quantity passing through the second one. The same conditions are required for the momentum fluxes.

1) The velocity component $u$ (tangential to the frontier) and the pressure $P$ are linearly interpolated.

2) The density $\rho$ is transmitted so that the quantity of mass $\rho S$ in each cell of the final row equals the sum of quantities $\rho_{i} S_{i}$ in the cells or part of cells of the original row (Fig. 7).

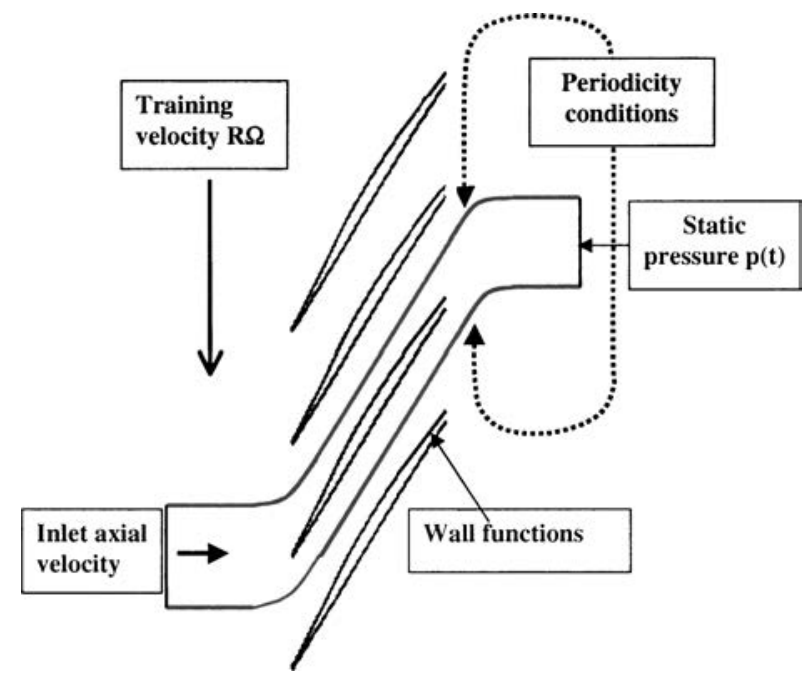

Fig. 9 Boundary conditions for a single-channel computation.

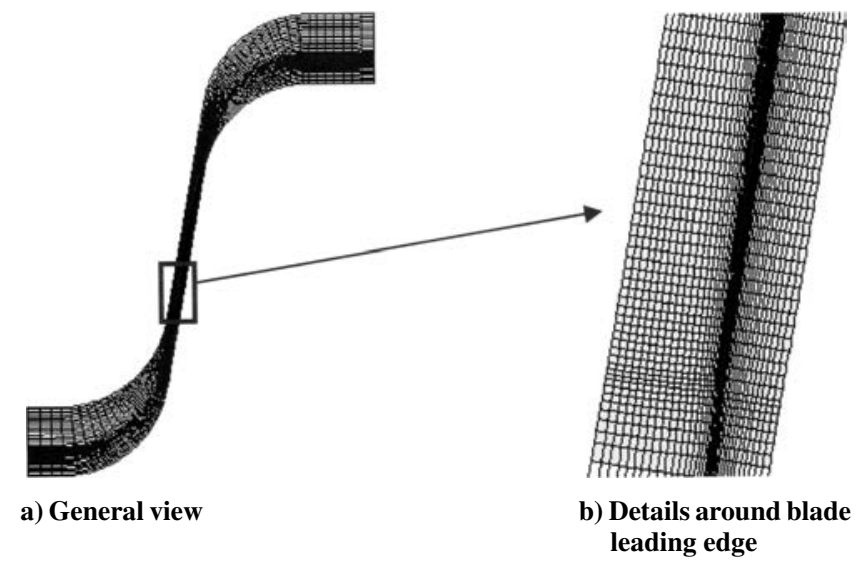

Fig. 10 Mesh of a single channel.
3) The transmission of the velocity component $v$ (normal to the frontier) is a little more complex because it must satisfy the conservation of both mass and momentum. Because staggered grids are used, $v$ is not located at the center of the cells, but on their northern and southern frontiers. As can be seen in Fig. 8a, $v$ is transmitted from lines $3^{\prime}, 4^{\prime}$ to lines $1^{\prime}, 2^{\prime}$ and from lines $1,2,3$ to lines $4,5,6$. Note that $v$ is calculated only on one of the two frontiers, here on line 1 , and the result is transmitted to the other one, line 4 . The procedure guarantees the equality of fluxes on these two lines, without altering the convergence rate. Unfortunately, mass and momentum conservations cannot be both obtained with only one variable $v$. To solve this problem, we took advantage of the specificity of the pressure correction algorithm, which is based on two separated resolutions of momentum and continuity equations.

\section{Integration in the Algorithm}

The velocities must be transmitted each time they are modified inside the computational domain, that is, two times per iteration: after the resolution of the momentum equations (step 1) and after the velocity correction involved in the pressure-correction method (step 2).

We considered that the first step was based on equilibrium between the momentum fluxes and the pressure gradients. Thus, the equality of the momentum fluxes on the connected frontiers must be verified during this step, and a B transfer (Fig. 8b) is performed just before. However, the second step is based on equilibrium between mass fluxes. These have to be equal on the two frontiers during this step, and an A transfer is performed immediately before.

\section{Application to a Two-Dimensional Blade Cascade}

The numerical model presented was applied to the calculation of the cavitating behavior of a four-blade cascade representing a complete rocket engine turbopump inducer. The objective was the simulation of the four sheets of cavitation attached to the blades and their unsteady coupled behavior.

\section{A. Single Channel Computation}

First only one blade-to-blade channel was considered. The uniform flow velocity imposed at the mesh inlet is deduced from the flow rate, and a periodicity condition is applied between the two sides of the channel (Fig. 9), according to the procedure presented in the preceding section. A uniform static pressure is imposed at the domain outlet, far enough from the trailing edge to avoid any influence of the boundary condition on the pressure field around the blade. Possible effects of the lines upstream and downstream from the inducer on its cavitating behavior are not taken into account in this approach.

A $190 \times 30$ structured mesh is used (Fig. 10). A special contraction of the grid is applied in the expected cavitating areas, that is, around the leading edge in the axial direction and on the suction side of the blade in the transverse direction. Along the solid boundaries, the $k-\varepsilon$ turbulence model is associated with standard laws of the wall, and so a grid contraction is applied on both sides of the blade

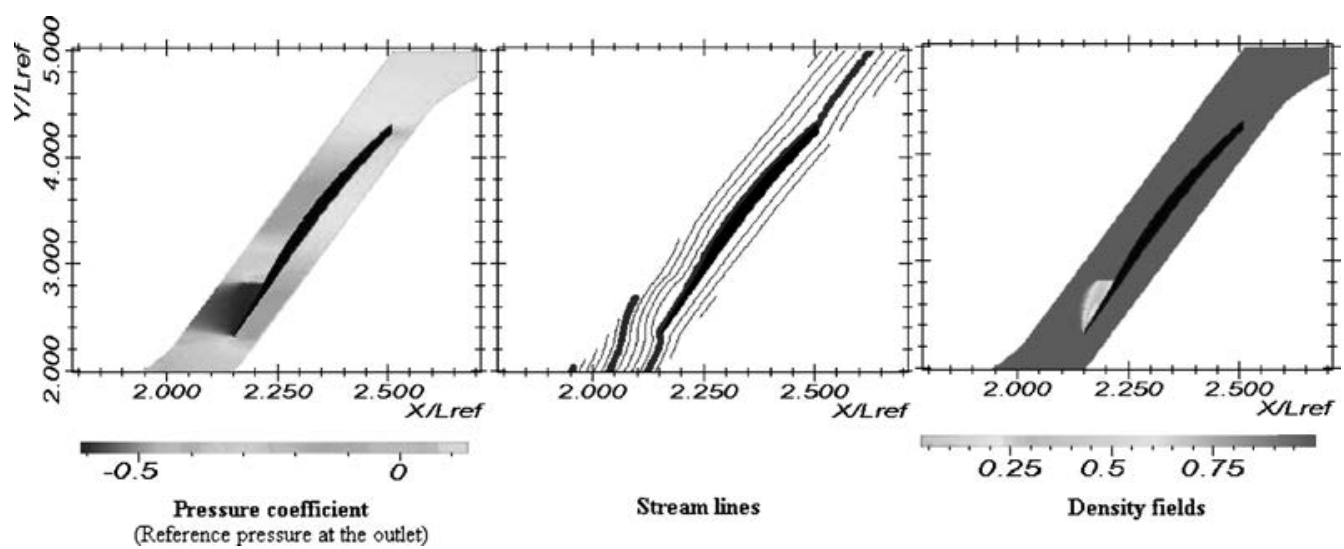

Fig. 11 Predicted cavitating flow in inducer bląde-to-blade channel $(\sigma=0.1$, nominal flow rate). 
to constrict $y+$ at the first grid point between 35 and 120 for the Reynolds value used. The study of the influence of the Reynolds number would need a remeshing near the blades surfaces and was not performed for that first application.

To characterize the flow around the blade, a slow decrease of the cavitation number is simulated. First, a stationary step is performed, which imposes the reference flow rate and an outlet static pressure high enough to avoid any presence of vapor in all of the computational domain. During the following time steps, this pressure is decreased progressively, and vapor appears on the blade suction side. Large time steps $\left(\Delta t=0.1 T_{\text {ref }}\right)$ are imposed during that slow transient, to minimize the time-dependent terms in the equations and to obtain quasi-steady flowfields. Figure 11 shows the flow characteristics obtained for a cavitation number equal to 0.1 . It can be seen that the shape of the sheet of cavitation is directly governed by the pressure field. The obstruction generated by the cavity in the flow also clearly appears on the streamlines representation. This obstruction increases when the cavitation number is still lowered, and it finally results in the drop of the blade performance, that is, an increase of the pressure inlet in the present case.

The entire behavior is recapitulated in Fig. 12, which shows the evolution of the blade performance as the cavitation number is lowered from initial noncavitating conditions down to the final performance drop. It consists successively in a quite stable evolution (for $\sigma>0.2$ ), a first pronounced drop at $\sigma=0.2$, and then a sudden increase of the head $(\sigma=0.1)$ just before the final blockage.

Although a large time step was used, the chart strongly fluctuates, which indicates that the cavitating flow is fundamentally unstable. Moreover, some numerical instability is observed when reaching the

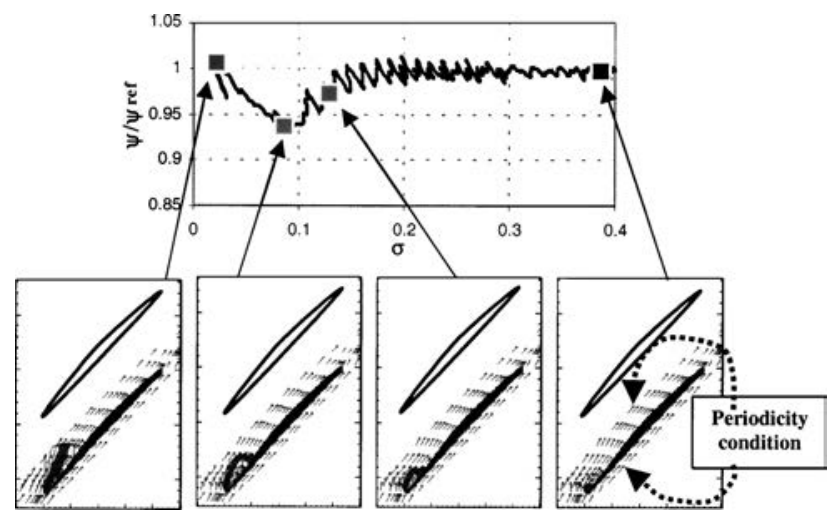

Fig. 12 Single-channel computation; cavitation characteristic of the cascade at $\phi=\phi_{\text {ref }}$ and associated length of the attached cavity (ratio 5:1 between horizontal and vertical scales). final head drop: The flow rate is imposed strictly at the inlet and the cavitation blockage is, thus, especially abrupt. The simulation was also performed with $\Delta t=0.025 T_{\text {ref }}$ and $\Delta t=0.4 T_{\text {ref }}$ to investigate the influence of the time step. The more $\Delta t$ is decreased, the more the sheet of cavitation fluctuates, but no noticeable difference can be observed for the mean performance evolution. The influence of the mesh on this mean performance was also tested ${ }^{23}$ with a finer grid composed of $220 \times 35$ cells, and less than $2 \%$ of difference with the standard grid was obtained in cavitating conditions $(\sigma<0.4)$.

The decrease/reincrease of the performance $(0.025<\sigma<0.1)$ was more closely investigated, to understand the mechanisms of this surprising behavior. Observation of the velocity fields indicates that this phenomenon is due the interaction between the attached sheet cavitation and the boundary layer on the blade: Figure 13 shows the modifications due to cavitation of the relative velocity $\boldsymbol{W}$ on the suction side of the blade. Velocities are reported in two sections located at midchord and at the trailing edge, respectively. Because of the blade orientation, the variations of $\boldsymbol{W}$ are almost consistent with the evolution of the tangential relative velocity $\boldsymbol{W}_{u}$.

The curves corresponding to $\sigma=0.12$ show a diminution of $\boldsymbol{W}$ inside the boundary layer, and an increase of $\boldsymbol{W}$ outside, compared with very low cavitating conditions $(\sigma=0.4)$. The predominant effect at this stage is the increase of $\boldsymbol{W}$ in the main part of the section, which leads to the progressive decrease of the blade performance. For $\sigma=0.08$, an opposite effect appears at midchord: $\boldsymbol{W}$ is increasing inside the boundary layer, while decreasing outside. Nevertheless, this effect is not strong enough to propagate to the trailing edge, where $\boldsymbol{W}$ is still globally increasing, which explains the important drop of the performance. For $\sigma=0.024$, it can be observed that the earlier effect has propagated all along the blade, and it was also amplified: $\boldsymbol{W}$ has much decreased in the major part of the sections (leading to a better pressure rise), although it notably increased close to the blade.

The progressive growth of the sheet of cavitation is responsible for these variations of $\boldsymbol{W}$. Figure 14a shows a scheme of this mechanism in the case of two consecutive blades, instead of the present periodicity condition. As $\sigma$ is lowered, the cavity width expands and the fluid at the blade leading edge is pushed upstream. This fluid is then moved by a part of the main flow toward the bottom of the channel and highly accelerated. It induces a jet effect on the suction side of the adjacent blade, which constricts the boundary layer closer to the blade. The result is an increase of the velocity close to the wall and a decrease in the other part of the section.

When $\sigma$ still decreases (Fig. 14b), this effect disappears because of the obstruction generated by the cavity: This explains the final performance drop.

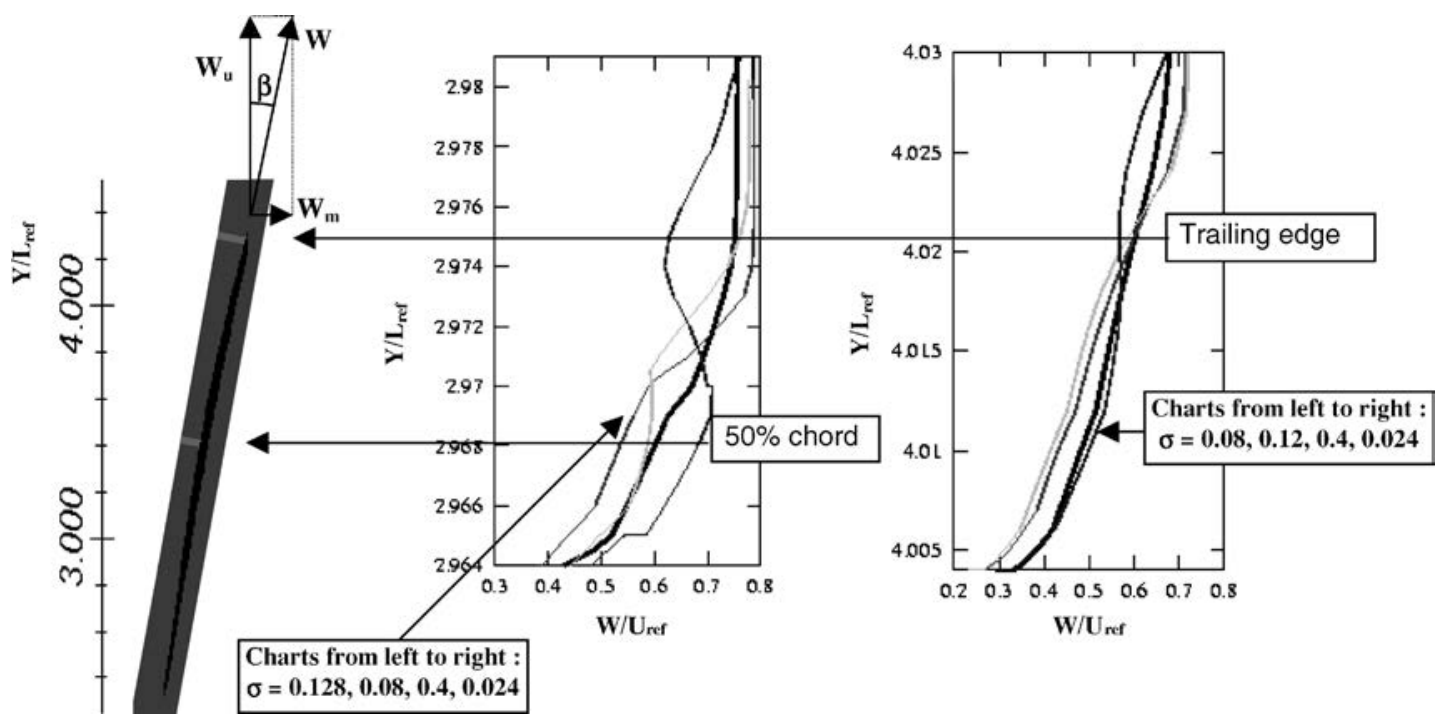

Fig. 13 Evolution of the relative velocity $W$ on blade suction side when cavitation number is decreased. 


\section{B. Four-Blade Cascade Computation: Steady Behavior}

Time-accurate computations are performed on the four-blade mesh composed of four connected earlier grids, that is, 28,000 cells. The periodicity condition is then applied between the fourth and first channels, as in the real runner. The time step was chosen to put emphasis on the investigation of the low-frequency fluctuations of the attached cavity, without being perturbed by the local unsteadiness in each cavitation sheet wake (cloud shedding): therefore, it is fixed equal to $1 \%$ of the blade passage time $T_{\text {ref }}$. Thus, the self-oscillation behavior of the cavities is not simulated. Successive time-accurate computations are performed at fixed cavitation number and nominal flow rate coefficient.

First cases with quite high cavitation number $(\sigma \approx 0.175)$ lead to stable and symmetrical small cavities attached on each blade (Fig. 15a). The coupling between the four channels does not generate in that case any supplementary unsteady effect: The four cavities remain identical, and their shape becomes constant in time after the initial transient, as in the single-channel computation. This behavior is shown in Fig. 15b, which presents the time evolution of the cavity in the first channel: It completely stabilizes after the initial fluctuations. (This transient from $t / T_{\text {ref }}=0$ to 10 corresponds to the growing of the attached cavity from the noncavitating initial condition.) Figure 16 shows only small oscillations in the mass flow rate through each passage. Therefore, in this configuration the four-channel coupling has only a very small effect on the cavitation unsteady behavior.

\section{Four-Blade Cascade Computation: Unsteady Behavior}

When the cavity length is increased $(\sigma=0.15)$, an unsteady configuration spontaneously appears. Figures 17 and 18 show that the

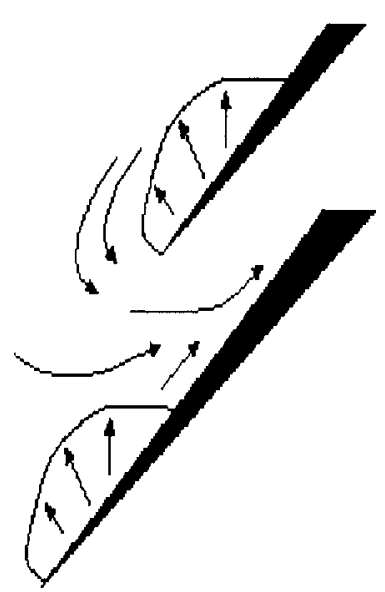

a) Increase for $0.03<\sigma<0.08$

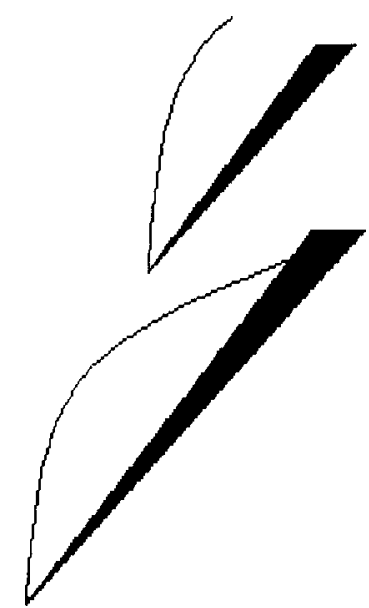

b) Final drop for $\sigma<0.02$
Fig. 14 Origin of performance variations. cavities are then different on the successive blades: three large cavities and a little cavity are simulteously obtained. This unbalanced cavitating structure propagates from blade to blade in time. That phenomenon takes place spontaneously, only from the very small perturbations due to numerical errors of truncation.

Figure 19 shows the repartition of the mass flow rate in the cascade. After the initial transient, periodic fluctuations take place with different phases in the four channels. Their amplitude increases when the cavitation number is slightly decreased from 0.15 to 0.135 . Visual observation (Fig. 17) and a fast Fourier transform analysis of cavity length fluctuations signal (Fig. 20) show that in the stator frame the phenomenon is about $50 \%$ faster than the inducer rotation speed. As observed in the experiments, this value slightly decreases with cavitation parameter. However, the numerical frequencies of that supersynchronous phenomenon remain about $25 \%$ larger than experimental values, as already observed in analytical models by Tsujimoto et al. ${ }^{10}$ and Joussellin and de Bernardi. ${ }^{11}$

For a lower cavitation parameter $(\sigma=0.08)$, a stable configuration appears (Fig. 21), with alternate long and short cavities on the blades and different flow rates in the successive channels. Such a configuration is observed experimentally but at a cavitation number higher than the supersynchronous range (Fig. 2).

Figure 22 shows the cavitating performance computed by averaging the head coefficient obtained with the four-channel simulations at fixed cavitation number and nominal flow rate coefficient (points $\triangle$ ). In the whole range of nonsymmetrical sheets of cavitation, the head coefficient is larger than the one obtained from the single-channel computation: the hollow in the chart is considerably reduced. This means that these special patterns of the flowfield (rotating supersynchronous cavitation and alternate cavitation) result in a diminution of the averaged losses in the blade cascade.

This effect can be interpreted on the basis of the analysis performed earlier in the case of the single-blade calculation. Indeed, rotating and alternate cavitation lead to the enlargement of some of the sheets of cavitation, compared to the stable configuration. The growth of these cavities pushes the fluid upstream at the leading edge. This fluid is then accelerated by the incoming flow, which results in the jet effect observed on the blade suction side in the adjacent channel (Sec. V.A). Thus, the flow in this channel is accelerated in the boundary layer, and decelerated above, which globally boosts the performance of the cascade. Nonsymmetrical flow patterns can, thus, be considered as a self-adaptation of the flow to reduce the losses in the blade-to-blade channels.

On the other hand, the final head drop is reached at higher $\sigma$ because of the lack of symmetry: The biggest of the cavities are larger than the one obtained in the case of a single-grid computation at the same cavitation number.

\section{Comparison with Experiments}

Quantitative comparison between the three-dimensional inducer and the two-dimensional blade cascade is not directly available

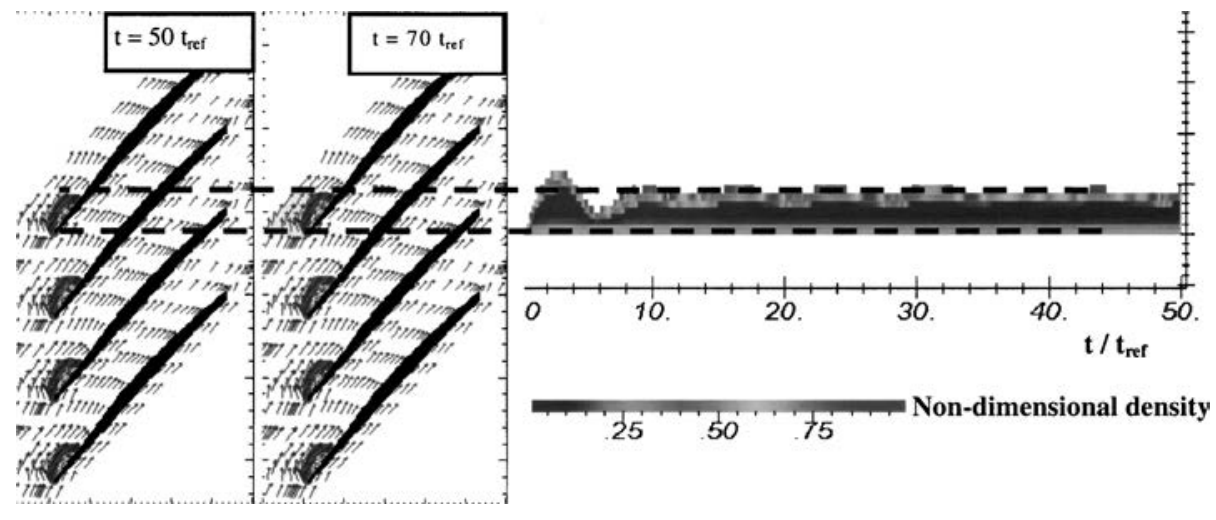

a)

b)

Fig. 15 Multiple channels computations: cavitation behavior at $\phi=\phi_{\text {ref }}$ and $\sigma \approx 0.1$ : a) cavitation pattern at $t=50 t_{\text {ref }}$ and $t=70 t_{\text {ref }}$ and b) time evolution of cavity length in the first channel, with time reported in abscissa, and $X$ position in the tunnel of cavitation graduated in ordinate. 
because of three-dimensional effects and the variation of the hub-toshroud ratio that is not taken into account. However, computations were performed at different operating conditions, by the varying of the cavitation number and the flow coefficient between $0.9 \Phi_{\text {ref }}$ and $1.2 \Phi_{\text {ref. }}$. The results are compared to experimental data in a qualitative way. The performance charts obtained are given in Fig. 23 with the limits of all nonsymmetrical flow arrangements, that is, rotating cavitation and alternate blade cavitation. For each flow rate, these limits are defined by two extreme values on a $\sigma$ scale, respectively $\sigma_{+}$and $\sigma_{-}$.

In all cases, the final head drop is predicted by the model at a too high $\sigma$ with respect to the experimental value (0.06 instead of 0.02$)$. The cavitation parameter range of rotating cavitation $\Delta \sigma=\left(\sigma_{+}-\sigma_{-}\right)$increases when the flow rate coefficient decreases. That result agrees with experimental observations performed by Pagnier et al. ${ }^{8}$ and Yokata et al. ${ }^{1}$

At reference flow coefficient $\phi_{\text {ref }}$, the experimental range of nonsymmetrical flow configurations is about $\Delta \sigma_{\text {exp }}=0.07$ (Fig. 2). Numerical results of $\Delta \sigma$ are plotted with respect to the flow rate coefficient in Table 1: The comparison shows that the experimental range corresponds to the numerical result at $\phi / \phi_{\text {ref }}$ between 1 and 1.05. That result can be explained qualitatively by an important difference in inlet conditions between experiments and the numerical prediction: In the experiments, a partial obstruction of the inlet section due to a backflow area in the vicinity of the casing is observed. This particular flow pattern is shown in Fig. 24 (see Ref. 24), with $S_{\text {flow }}$ indicating a whole cross section, $S_{\text {back }}$ the size of the backflow, and $S_{\text {cav }}$ the area occupied by cavitation near the shroud.

Table 1 Instability range vs flow coefficient

\begin{tabular}{ll}
\hline \hline$\phi / \phi_{\text {ref }}$ & $\Delta \sigma$ \\
\hline 0.90 & 0.16 \\
0.95 & 0.125 \\
1.0 & 0.08 \\
1.05 & 0.065 \\
1.1 & 0.04 \\
1.2 & 0 \\
\hline \hline
\end{tabular}

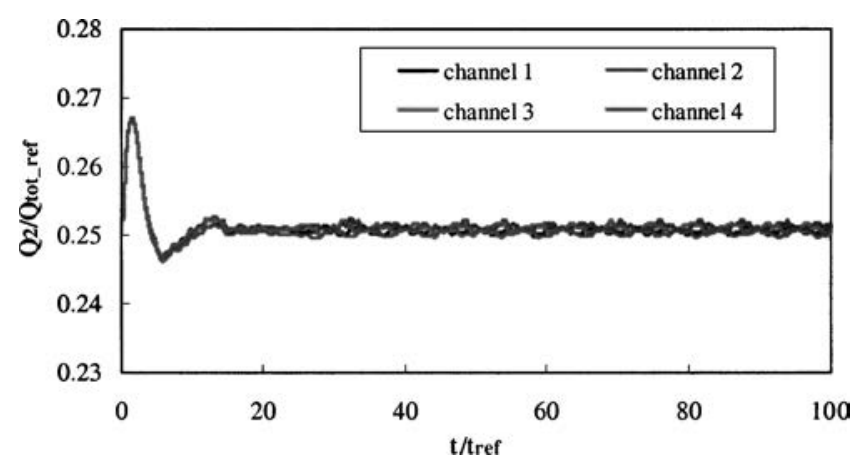

Fig. 16 Mass flow rate repartition in the four channels; $\sigma=0.175$.
This phenomenon is not taken into account in the present simulations, which modifies the cascade inlet condition: In experiments at nominal flow rate the obstruction leads to a higher component $\boldsymbol{C}_{m}$ of the velocity than in the simulation. For a higher simulated flow rate, inlet conditions are recovered, and $\Delta \sigma$ is consistent with $\Delta \sigma_{\text {exp }}$.

A comparison between the experimental breakdown chart at nominal flow rate and the one obtained by the four-channel computation is presented in Fig. 25. Although the present model, mainly because of the passage from three dimensional to two dimensional, cannot precisely quantify the location of breakdown and instability, a general correct agreement is obtained with experiments.

\section{E. Instability Criterion}

The evolution of the instability range according to the mass flow rate was investigated, to improve the understanding of the mechanisms that govern the onset and the conclusion of nonsymmetrical flow pattern.

Noted in Fig. 23 that the ending of instability occurs just at the beginning of the final drop of the cascade performance. Indeed, it has already been said (Sec. V.A) that this drop was due to the obstruction of the blade-to-blade channels by the sheets of cavitation. Thus, a critical size of the cavities could be directly responsible for the conclusion of the alternate blade cavitation. This assumption is confirmed in Fig. 26a, which presents the same six head drop charts as functions of the classical parameter $\sigma / 2 \alpha$, which is usually considered as the key parameter to determine the

Channel 1

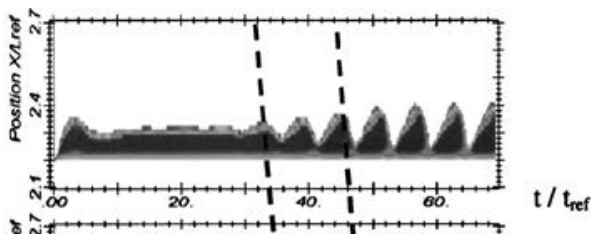

Channel 2

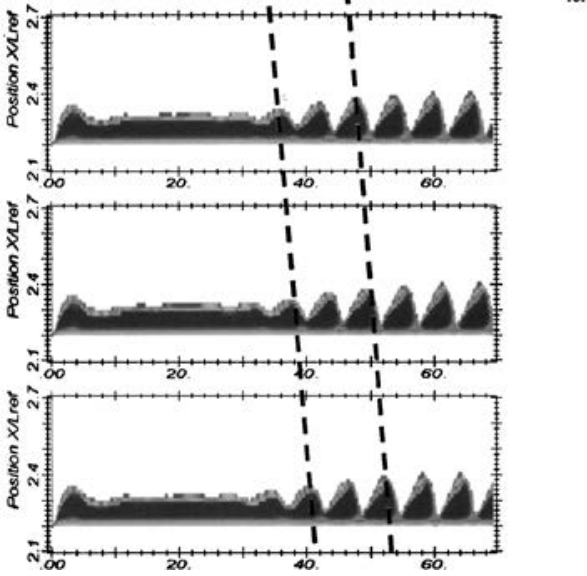

Fig. 18 Cavity length evolution on the four blades: amplification of the unsteady coupling and phase shift $(\sigma=0.15)$.
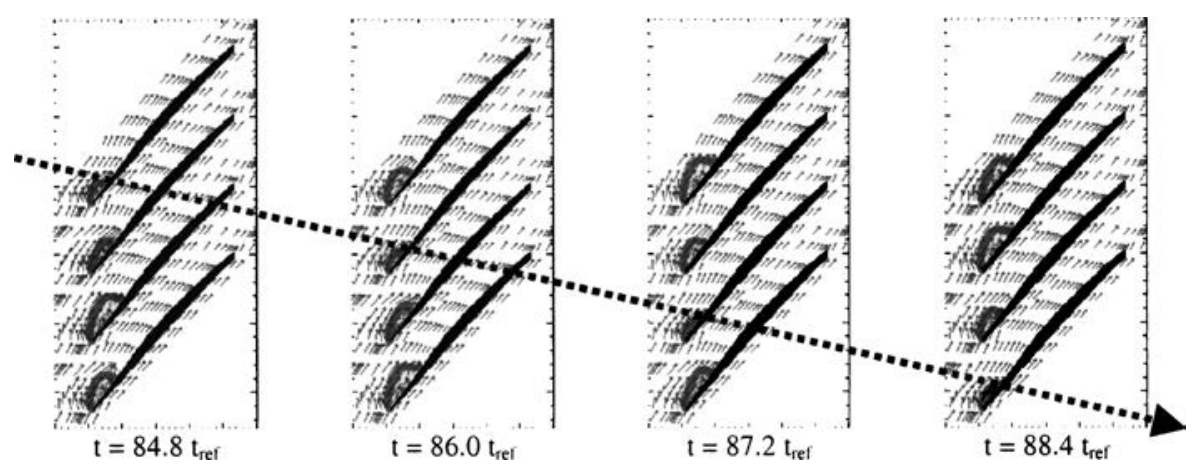

Fig. 17 Cavitation behavior at $\phi=\phi_{\text {ref }}$ and $\sigma=0.15$. 

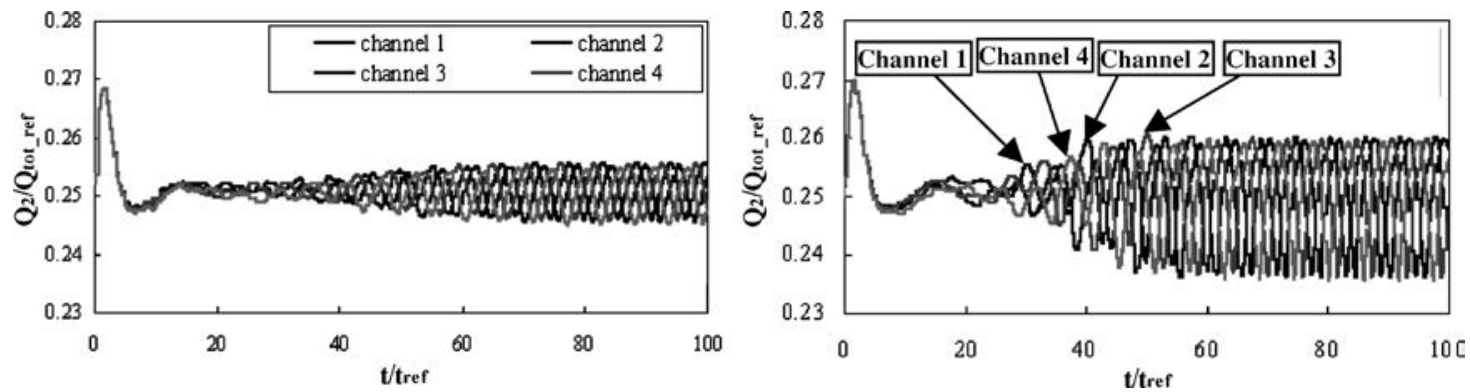

a) $\sigma=0.15$

b) $\sigma=\mathbf{0 . 1 3 5}$

Fig. 19 Flow rate repartition in the four channels; $\phi=\phi_{\text {ref. }}$.

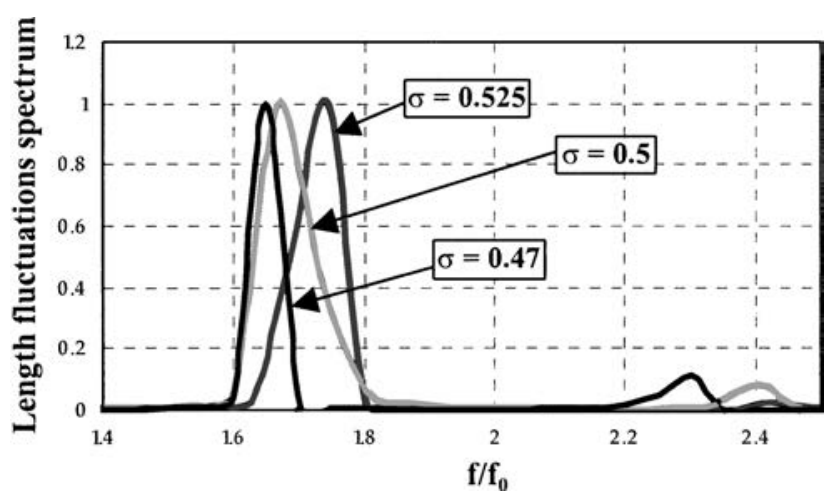

Fig. 20 Spectral analysis of the cavity fluctuations in the static frame.

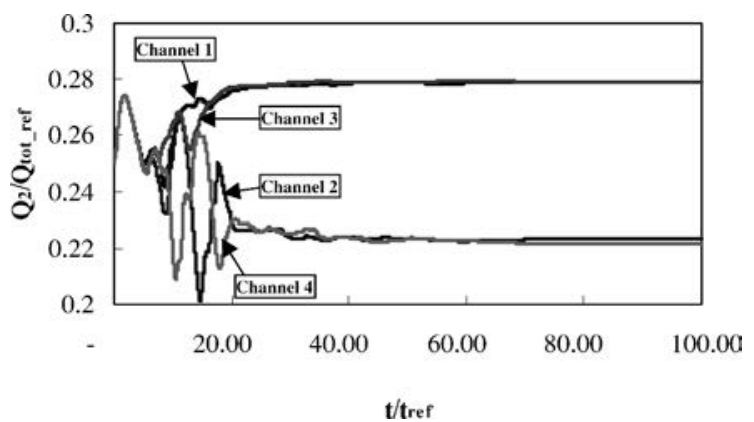

Fig. 21 Flow rate repartition in the four channels; $\phi=\phi_{\text {ref }}, \sigma=0.08$.

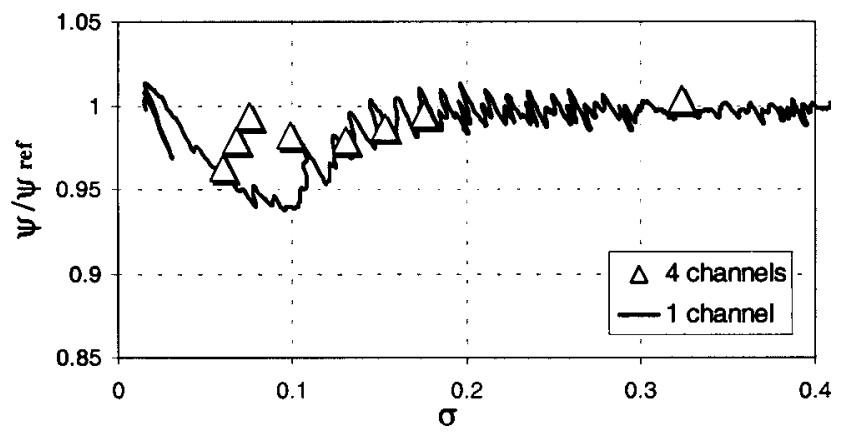

Fig. 22 Cavitation characteristic of the cascade: comparison between single-channel and four-channels computations; $\phi=\phi_{\text {ref }}$.

length of the cavity in a given configuration. For all of the mass flow rates, instability vanishes for $\sigma / 2 \alpha \approx 0.5 / 0.6$. This value is fully consistent with the criterion obtained by Tsujimoto et al. ${ }^{25}$ in the case of experimental results obtained with a three-blade inducer.

With regard to the onset of instability, this criterion cannot be applied, as can be seen in Fig. 26a. Indeed, according to the model, rotating cavitation appears earlier, that is, for a smaller length of

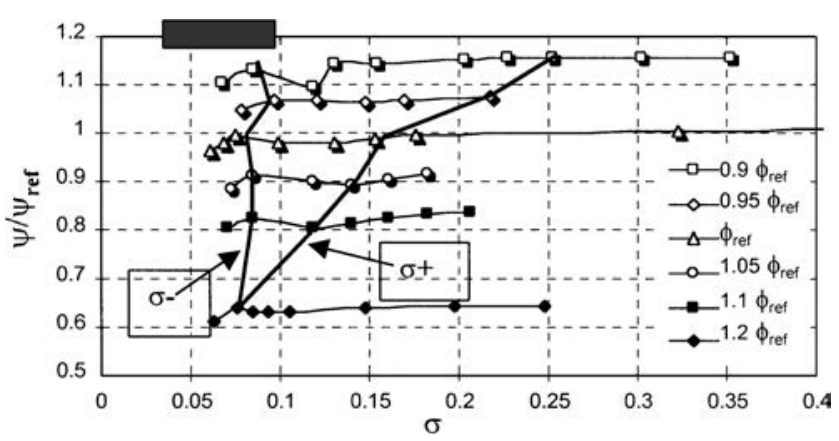

Fig. 23 Effect of the flow rate on the cavitation characteristic and on the instability range: - limit of nonsymmetrical arrangements and -, experimental instability range $\Phi=\Phi_{\text {ref }}$.

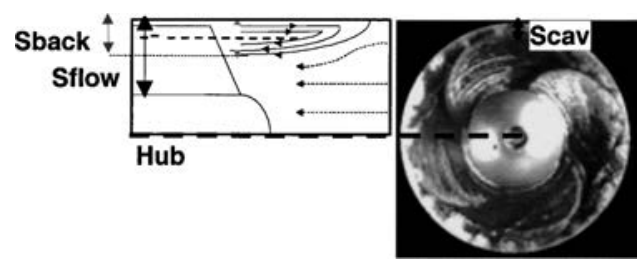

a)

b)

Fig. 24 Inducer inlet backflow ${ }^{24}$ : a) scheme of backflow area upstream from the blades and b) corresponding axial view of the inducer inlet.

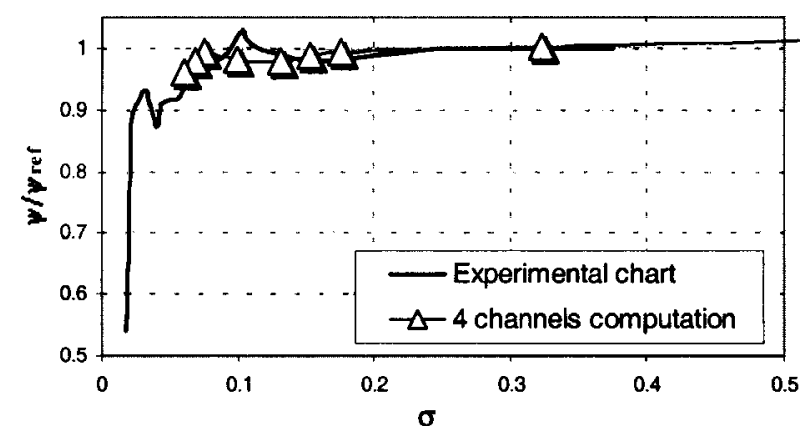

Fig. 25 Comparison between the experimental performance chart and the result of the four-channel computation at nominal flow coefficient.

cavitation sheets, when the mass flow rate decreases. This effect could be related to the increase of angle of attack, which enhances the flow separation at the blade leading edge and, thus, increases the obstruction generated by cavities, even for very small cavities. Thus, the inception of rotating cavitation would allow reducing the losses, as noted in Fig. 22. This additional influence of the flow incidence at the leading edge is confirmed by the instability map as a function of $\sigma / \alpha^{3}$ (Fig. 26b). An almost constant value of $\sigma / \alpha^{3}=350$ is obtained, which could be a criterion for instability inception in blade-to-blade calculations. 

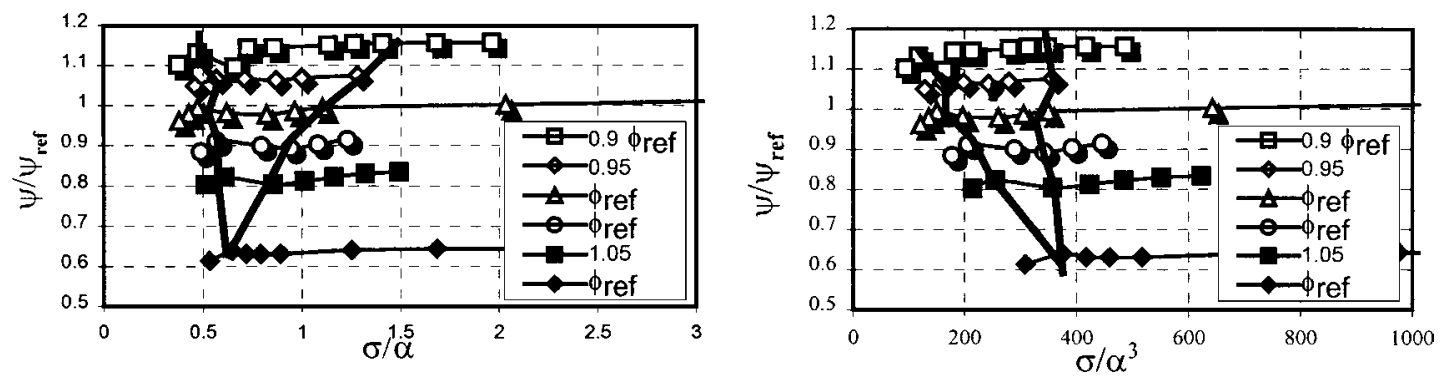

Fig. 26 Limit of nonsymmetrical flow patterns according to $\sigma / \alpha$ and $\sigma / \alpha^{3}$.

\section{Conclusions}

We have presented two-dimensional computations performed on a four-blade cascade geometry representative of the behavior of a real three-dimensional inducer. This work implied the development of a nonmatching connection treatment, based on an interpolation process adapted to the SIMPLE algorithm. Attention was focused on the study of nonsymmetrical flow patterns that occur in inducers, that is, rotating supersynchronous cavitation and alternate cavitation. These two flow configurations were successfully predicted by the numerical model at several mass flow rate conditions. Comparisons between single-channel and four-channel computations revealed that nonsymmetrical flow patterns allow suppressing the hollow in the blade cascade performance before the final breakdown. The analysis of the velocity field on the blade suction side in the case of a single-blade computation suggests that this reduction of the losses could be linked to an important acceleration of the flow in the channels where the cavity is small. This jet effect modifies the boundary layer on the blade suction side, which globally leads to a decrease of the losses for the whole cascade. Instability criteria independent from the flow rate were found, for instability inception and conclusion.

This work is pursued to clarify the effects of rotation speed and fluid parameters and to assess the prediction capability of the model. We intend more particularly to take into account the mentioned three-dimensional effects. Therefore, a full threedimensional model is developed to predict cavitating flowfields in inducers. The final objective is to apply the numerical method presented in the present paper to three-dimensional computations, to predict the unsteady effects associated with cavitation in the real three-dimensional geometry.

\section{Acknowledgments}

The authors thank SNECMA Moteurs (Rocket Engine Division) and the French space agency Centre National d'Etudes Spatiales for their support of the present work. We are also grateful to the CREMHyG laboratory (Grenoble, France), who provided the experimental results.

\section{References}

${ }^{1}$ Yokata, K., Kurahara, K., Kataoka, D., Tsujimoto, Y., and Acosta, A. J., "A Study on Swirling Backflow and Vortex Structures at the inlet of Inducer," Japan Society of Mechanical Engineers International Journal, Series B, Vol. 42-3, 1999, pp. 451-459.

${ }^{2}$ Laborde, R., Chantrel, P., Retailleau, A., Mory, M., and Boulon, O., "Tip Clearance Cavitation in an Axial Flow Pump," Proceedings of Cav'95 International Symposium, May 1995.

${ }^{3}$ Stutz, B., and Reboud, J. L., "Experiments on Unsteady Cavitation," Experiments in Fluids, Vol. 22, 1997, pp. 191-198.

${ }^{4}$ Kubota, A., Kato, H., and Yamagushi, H., "A New Modelling of Cavitation Flows: A Numerical Study of Unsteady Cavitation on a Hydrofoil Section," Journal of Fluid Mechanics, Vol. 240, 1992, pp. 59-96.

${ }^{5}$ Chen, Y., and Heister, S. D., "Modeling Hydrodynamic Nonequilibrium in Cavitating Flows," Journal of Fluids Engineering, Vol. 118, 1996, pp. 172-178.

${ }^{6}$ Kamijo, K., Shimura, T., and Watanabe, M., "An Experimental Investigation of Cavitating Inducer Instability," American Society of Mechanical Engineers, ASME Paper 77-WA/FW-14, 1977.

${ }^{7}$ de Bernardi, J., Joussellin, F., and Von Kaenel, A., "Experimental Analysis of Instabilities Related to Cavitation in Turbopump Inducer," Proceedings of the 1st International Symposium on Pump Noise and Vibrations, 1993, pp. 91-99.
${ }^{8}$ Pagnier, P., Morel, P., Spettel, F., Henry, C., and Champagne, J. Y., "Conception and Experimental Study of an Inducer," Proceedings of Cav'95 International Symposium, May 1995.

${ }^{9}$ Goirand, B., Mertz, A., Joussellin, F., and Rebattet, C., "Experimental Investigation of Radial Loads Induced by Partial Cavitation with Liquid Hydrogen Induce," Proceedings of the 3rd International Conference on Cavitation, ImechE, 1992, pp. 263-269.

${ }^{10}$ Tsujimoto, Y., Kamijo, K., and Yoshida, Y., "A Theoretical Analysis of Rotating Cavitation Inducers," Journal of Fluids Engineering, Vol. 115, 1993, pp. 135-141.

${ }_{11}$ Joussellin, F., and de Bernardi, J., "Analytical Modelling of Instabilities in a Cavitating Turbopump Inducer," Proceedings of the 2nd International Symposium on Cavitation, 1994, pp. 98-94.

${ }^{12}$ Pilipenko, V., Semyonov, Y., and Kvasha, Y., "Theoretical and Experimental Computational Methods for Determining Volume of Cavities and Coefficients of Dynamic Equation of Cavitation Cavities," TR SEP TC/T 23227/96, 1995 .

${ }^{13}$ Watanabe, S., Tsujimoto, Y., Franc, J. P., and Michel, J. M., "Linear Analyses of Cavitation Instabilities," Proceedings of the 3rd International Symposium on Cavitation, edited by J.-M. Michel and H. Kato, 1998, pp. 347-352.

${ }^{14}$ Reboud, J. L., and Delannoy, Y., "Two-Phase Flow Modeling of Unsteady Cavitation," Proceedings of the 2nd International Symposium on Cavitation, 1994.

${ }^{15}$ Reboud, J. L., Stutz, B., and Coutier, O., "Two Phase Flow Structure of Cavitation: Experiment and Modeling of Unsteady Effects," Proceedings of the 3rd International Symposium on Cavitation, edited by J.-M. Michel and H. Kato, 1998, pp. 225-232.

${ }^{16}$ Coutier-Delgosha, O., Reboud, J. L., and Delannoy, Y., "Numerical Simulations in Unsteady Cavitating Flows," International Journal for $\mathrm{Nu}$ merical Methods in Fluids, Vol. 42, No. 5, 2003, pp. 527-548.

${ }^{17}$ Lohrberg, H., Stoffel, B., Fortes-Patella, R., Coutier-Delgosha, O., and Reboud, J. L., "Numerical and Experimental Investigation on the Cavitating Flow in a Cascade of Hydrofoils," Experiments in Fluids, Vol. 33/4, 2002, pp. 578-586.

${ }^{18}$ Delannoy, Y., and Kueny, J. L., "Two Phase Flow Approach in Unsteady Cavitation Modeling," Proceedings of the Cavitation and Multiphase Flow Forum, ASME-FED, Vol. 98, American Society of Mechanical Engineers, Fairfield, NJ, 1990, pp. 153-158.

${ }^{19}$ Merkle, C. L., Feng, J., and Buelow, P. E. O., "Computational Modeling of the Dynamics of Sheet Cavitation," Proceedings of the 3rd International Symposium, on Cavitation, Vol. 2, edited by J.-M. Michel and H. Kato, 1998, pp. 307-314.

${ }^{20}$ Kunz, R., Boger, D., Chyczewski, T., Stinebring, D., and Gibeling, H., "Multi-Phase CFD Analysis of Natural and Ventilated Cavitation About Submerged Bodies," Proceedings of the 3rd ASME/JSME Joint Fluids Engineering Conference, July 1999.

${ }^{21}$ Zhu, J., "A Low Diffusive and Oscillation-Free Convection Scheme," Communications in Applied Numerical Methods, Vol. 7, 1991, pp. 225-232.

${ }^{22}$ Coutier-Delgosha, O., Fortes-Patella, R., and Reboud, J. L., "Evaluation of the Turbulence Model Influence on the Numerical Simulations of Unsteady Cavitation," Journal of Fluids Engineering, Vol. 125, 2003, pp. 38-45.

${ }^{23}$ Courtot, Y., "Instabilités de Cavitation dans les Inducteurs: Analyse et Modélisation de l'Écoulement Instationnaire en Grilles d'Aubes," Ph.D. Dissertation, Institut National Polytechnique de Grenoble, Grenoble, France, Dec. 2000.

${ }^{24}$ Joussellin, F., Courtot, Y., Coutier-Delgosha, O., and Reboud, J. L., "Cavitating Inducer Instabilities: Experimental Analysis and 2D Numerical Simulation of Unsteady Flow in Blade Cascade," Proceedings of the 4th International Symposium on Cavitation, edited by R. E. A. Arndt, C. E. Brennen, and S. Ceccio, June 2001.

${ }^{25}$ Tsujimoto, Y., Watanabe, S., and Horiguchi, H., "Linear Analysis of Cavitation Instabilities of Hydrofoils and Cascade," Proceedings of USJapan Seminar: Abnormal Flow Phenomena in Turbomachinery, 1998. 